\title{
Scaling laws for edge plasma parameters in ITER from two-dimensional edge modelling
}

\author{
A.S. Kukushkin ${ }^{1}$, H.D. Pacher ${ }^{2}$, G.W. Pacher ${ }^{3}$, G. Janeschitz ${ }^{4}$, \\ D. Coster ${ }^{5}$, A. Loarte $^{6}$ and D. Reiter \\ ${ }^{1}$ ITER IT, Boltzmannstr. 2, 85748 Garching, Germany \\ 2 INRS-EMT, Varennes, Québec, Canada \\ ${ }^{3}$ Hydro-Québec, Varennes, Québec, Canada \\ ${ }^{4}$ FZK-PL-Fusion, Karlsruhe, Germany \\ ${ }^{5}$ Max-Planck Institut für Plasmaphysik, Garching, Germany \\ ${ }^{6}$ EFDA, Garching, Germany \\ ${ }^{7}$ FZ Jülich, Jülich, Germany \\ E-mail: kukusha@itereu.de
}

Received 14 November 2002, accepted for publication 27 June 2003

Published 1 August 2003

Online at stacks.iop.org/NF/43/716

\begin{abstract}
Results of a detailed study of the parameter space of the ITER divertor with the B2-Eirene code are presented. Relations between plasma parameters at the separatrix, the interface between the core and edge plasma, are parametrized to provide a set of boundary conditions for the core models. The reference ITER divertor geometry is compared with the straight target option, and the possibility of controlling the edge density by shifting the plasma equilibrium in ITER is explored.
\end{abstract}

PACS numbers: 28.52.Av, 52.55.Rk, 52.65.W

\section{Introduction}

Recent studies [1-4] have shown that there is an operational window for which the ITER divertor is expected to provide both acceptable target loading and the required efficiency of helium ash removal. This paper is devoted to a further exploration of the parameter space of the ITER divertor with major emphasis on the consistency of the divertor operational window with the required core plasma performance. The rationale for these studies is described below.

The upstream plasma density saturates with an increase in the fuelling rate $[3,4]$, thus limiting the operational window to rather low plasma density at the separatrix, $n_{\mathrm{s}} \sim(3-4) \times 10^{19} \mathrm{~m}^{-3}$, which is consistent with experimental indications that good plasma confinement in the H-mode requires low separatrix density [5]. To produce sufficient fusion power and a sufficiently high fusion gain, $Q$, the average plasma density in ITER must be $(8-10) \times 10^{19} \mathrm{~m}^{-3}$ with a flat density profile in the centre. Consequently, a significant density gradient must be sustained in the 'pedestal' region just inside the separatrix, and thus considerable particle fluxes must traverse this region $[3,6]$. Since the neutral particle influx across the separatrix also saturates at a comparatively low level [3,4], an additional fuelling scheme such as pellet injection has to be employed. This results in a core fuelling rate comparable to, or higher than, the gas puffing rate [7,8], whereas most of the calculations [1-4] have been done for conditions of predominant gas fuelling. A difference in the mechanisms of energy transport by electrons and ions in the plasma core (different heat conductivities, different heating power, different radiation and charge exchange losses) leads to unequal power transferred by these plasma components across the separatrix, $P_{\mathrm{e}}$ and $P_{\mathrm{i}},[7,8]$ whereas $P_{\mathrm{i}}=P_{\mathrm{e}}$ had been assumed in [1-4]. Furthermore, only conditions of predominant gas fuelling in the reference ITER divertor geometry with a V-shaped target and the separatrix strike point near the vertex were considered in $[3,4]$ where a wellpronounced saturation of the upstream density was found. If the saturation were caused by this special geometrical arrangement, then moving the separatrix strike point upward would allow higher $n_{\mathrm{s}}$, adding to the operational flexibility of the machine.

Following these lines, we discuss the interplay between the core and edge plasma in section 2 , analyse the effect of the 
partition of power between species and of the fuelling source in section 3, present the fitting formulae and coefficients for the interface plasma parameters in section 4 , and explore the potential of the separatrix density control through a variation of the strike-point position in section 5. In addition, the effect of toroidal geometry in modelling the neutral particle transport in the divertor and scrape-off layer (SOL) is explored in section 6, and conclusions are given in section 7.

\section{Core-edge interplay}

Consistency of the edge plasma parameters with the core can be ensured if one solves the equations for the edge plasma and for the core plasma simultaneously, matching the densities, temperatures, and fluxes at the separatrix. Given the different timescales and models for the core (hundreds of seconds, close to one-dimensional) and edge (tens of milliseconds, twodimensional), it is presently impractical to couple the codes directly. Instead, the solutions for the edge are parametrized in terms of the variables resulting from core simulations, and the output quantities from the two-dimensional simulations are used as boundary conditions for the 1-1/2-dimensional core plasma code ASTRA [7, 8].

The natural choice for the edge input parameters is the input power to the SOL $P_{\mathrm{SOL}}$, the pumping speed $S_{\mathrm{DT}}$, the $\mathrm{D} / \mathrm{T}$ particle throughput $\Gamma_{\mathrm{DT}}$, the fraction of the throughput supplied by core fuelling $\eta_{\mathrm{c}}=\Gamma_{\text {core }} / \Gamma_{\mathrm{DT}}$, the ratio of power input in the electron and ion channels $\xi_{\mathrm{ei}}=P_{\mathrm{e}} / P_{\mathrm{i}}$, and the helium ion flux across the separatrix, which is determined by the fusion power and the helium atom influx into the core. The output quantities, which serve as the boundary conditions for the core plasma, are the separatrix-averaged values of the electron and ion temperatures $T_{\mathrm{e} \_ \text {sep }}$ and $T_{\mathrm{i} \text { sep }}$, and electron, $\mathrm{He}$, and $\mathrm{C}$ densities, $n_{\mathrm{sep}}, n_{\mathrm{He} \_s e p}$, and $n_{\mathrm{C} \_s e p}$, together with $\mathrm{D} / \mathrm{T}$ and He neutral outfluxes from the edge to the core $\Gamma_{\mathrm{DT} \_n \_s e p}$ and $\Gamma_{\text {He_n_sep }}$, and the mean energy of these neutrals $E_{\text {DT_sep }}$ and

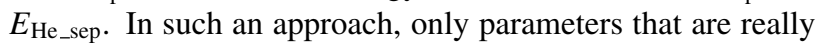
controllable in experiment, namely auxiliary heating power, pumping speed, gas puffing rate, and core fuelling rate (e.g. pellets, beams), are used as control parameters in modelling. In addition, the peak power loading of the target $q_{\mathrm{pk}}$ is used to constrain the operational window of the core plasma.

The importance of $n_{\mathrm{He} \_s e p}$ and $\Gamma_{\mathrm{He} \_ \text {_s sep }}$ can easily be seen from the following consideration (a generalization of that in [9]). Relying on diffusive helium transport in the plasma core, one can write the equation for the steady-state helium ion density from the plasma centre to the separatrix as

$$
\operatorname{div} j_{\mathrm{He}}=S_{\alpha}+S_{\text {rcy }}
$$

where

$$
j_{\mathrm{He}}=-D_{\mathrm{He}} \frac{\mathrm{d} n_{\mathrm{He}}}{\mathrm{d} r}+v_{\mathrm{A}} n_{\mathrm{He}}
$$

and the helium ion source consists of the fusion source $S_{\alpha}$ and ionization of recycled helium atoms inside the separatrix $S_{\text {rcy }}\left(\int S_{\text {rcy }} \mathrm{d} V=\Gamma_{\text {He_n_sep }}\right)$. The boundary condition at the separatrix is

$$
\left.n_{\mathrm{He}}\right|_{r=a}=n_{\text {He_sep }}
$$

Since the helium transport is practically linear (helium concentration is low, so that the helium-helium collisions together with the He contribution to the energy balance can be neglected and helium is unlikely to affect the anomalous, presumably turbulent, transport), the solution to equation (1) can be written as

$$
\begin{gathered}
n_{\mathrm{He}}=n_{\mathrm{He}}^{(1)}+n_{\mathrm{He}}^{(2)}+n_{\mathrm{He}}^{(3)} \\
\operatorname{div} j_{\mathrm{He}}^{(1)}=S_{\alpha},\left.\quad n_{\mathrm{He}}^{(1)}\right|_{r=a}=0 \\
\operatorname{div} j_{\mathrm{He}}^{(2)}=S_{\mathrm{rcy}},\left.\quad n_{\mathrm{He}}^{(2)}\right|_{r=a}=0 \\
\operatorname{div} j_{\mathrm{He}}^{(3)}=0,\left.\quad n_{\mathrm{He}}^{(3)}\right|_{r=a}=n_{\mathrm{He} \_s e p}
\end{gathered}
$$

By definition, the effective particle confinement time for helium is

$$
\tau_{\mathrm{He}}^{*}=\frac{\left\langle n_{\mathrm{He}}\right\rangle V}{S_{\alpha}}
$$

( $\left\langle n_{\mathrm{He}}\right\rangle$ means volume-average value, $V$ is the plasma volume). Using equation (3), one can obtain

$$
\tau_{\mathrm{He}}^{*}=\tau_{\mathrm{He}}+\tau_{\mathrm{He}}^{(2)}+\tau_{\mathrm{He}}^{(3)}
$$

where

$$
\begin{gathered}
\tau_{\mathrm{He}}=\frac{\left\langle n_{\mathrm{He}}^{(1)}\right\rangle V}{\int S_{\alpha} \mathrm{d} V}, \quad \tau_{\mathrm{He}}^{(2)}=\frac{\left\langle n_{\mathrm{He}}^{(2)}\right\rangle V}{\int S_{\alpha} \mathrm{d} V}, \\
\tau_{\mathrm{He}}^{(3)}=\frac{\left\langle n_{\mathrm{He}}^{(3)}\right\rangle V}{\int S_{\alpha} \mathrm{d} V}
\end{gathered}
$$

Therefore, $\tau_{\mathrm{He}}^{*}$ is a sum of three components. The first is determined by the helium transport inside the plasma core and does not depend on the pumping conditions, and the last two are proportional to $n_{\mathrm{He} \_s e p}$ and $\Gamma_{\mathrm{He} \_n \_s e p}$, respectively.

To estimate their importance for the case of ITER, we performed a series of ASTRA calculations using the formalism of $[7,8]$ and anticipating the scaling relations of section 4 . It turns out that, for ITER conditions, the edge helium density contributes $\frac{1}{4}$ to $\frac{1}{3}$ to the ratio of helium pedestal density to helium average density and to $\tau_{\mathrm{He}}^{*} / \tau_{E}$. The helium neutrals contribute $\frac{1}{3}$ to $\frac{1}{2}$ at low electron density $\left(0.7 \times 10^{20} \mathrm{~m}^{-3}\right)$ and only 5-7\% at high electron density $\left(1.4 \times 10^{20} \mathrm{~m}^{-3}\right)$. Therefore the part of $\tau_{\mathrm{He}}^{*} / \tau_{E}$ that is affected by pumping and which can, in principle, be reduced by improvements in pumping, i.e. the sum of these two terms, varies from $\frac{2}{3}$ at low density to $\frac{1}{2}$ at normal density to $\frac{1}{3}$ at high density. $\tau_{\mathrm{He}}^{*} / \tau_{E}$ itself is $2-4$ with standard pumping, and would become $\tau_{\mathrm{He}} / \tau_{E} \cong 1.3$ if the pumping (and the D/T throughput) could be increased by a large factor.

\section{Effect of the partition of power between species and of the fuelling source}

A first parametrization of separatrix parameters for use in core modelling was presented in [4] where the cases considered were mostly gas-fuelled and had $\xi_{\text {ei }}=1$. It was shown that a two-regime power law scaling could be constructed, which fitted the output data discussed in the previous section $\left(q_{\mathrm{pk}}\right.$, $\left.n_{\text {sep }}, n_{\mathrm{He} \_ \text {sep }}\right)$ quite well. The point where the density started to saturate for gas-puffed cases (and near which the inner divertor detached) was found to delimit the two regimes of divertor operation having different exponents in the power law scalings. 
The input parameter space was essentially three-dimensional $\left(P_{\mathrm{SOL}}, \Gamma_{\mathrm{DT}}, S_{\mathrm{DT}}\right)$ : no data were yet available for the $\xi_{\mathrm{ei}}$ variation and only a few points were available for core-fuelled cases $\left(\eta_{\mathrm{c}}\right.$ variation). Taking these variations into account increases the dimensionality of the input parameter space from three to five, which makes full coverage of the parameter space in two-dimensional calculations rather unwieldy. Therefore, we will concentrate here on the effect of these two new variables, having fixed $P_{\mathrm{SOL}}=100 \mathrm{MW}$ and $S_{\mathrm{DT}}=20 \mathrm{~m}^{3} \mathrm{~s}^{-1}$ for the newer simulations, and use the scalings obtained in [4] to parametrize the solution in $P_{\mathrm{SOL}}$ and $S_{\mathrm{DT}}$.

All the modelling results presented here are obtained with the B2-Eirene code package $[10,11]$ based on a twodimensional multi-fluid description for electrons and ions coupled to a Monte-Carlo model for the neutral components (atoms and molecules), with special modifications aimed at improvement of the accuracy of the particle balance [3]. No fast transients such as ELMs are present in the model. The results should therefore be considered as time-averaged data forming a basic level which is perturbed by ELMs. Such an approach can be justified by noting that in ITER only some $10 \%$ of the average power is expected to be transferred by ELMs [12], and by the fact that the modelling region in the two-dimensional calculations here does not include the pedestal. Drifts are also not included, and the divertor asymmetry ( $\sim 2: 1$ for outer divertor in terms of power) is ensured by the Shafranov shift of the plasma equilibrium which makes the gradients and thus the radial flows stronger on the outboard side of the torus. The B2-Eirene modelling conditions are the same as in [4]. In particular, the plasma consists of D (representing both $\mathrm{D}$ and $\mathrm{T}$ ), He, and $\mathrm{C}$ ions, no external impurity injection (impurity seeding) is applied, carbon is sputtered from the targets and absorbed everywhere on the surfaces, and elastic collisions of $\mathrm{He}$ atoms with $\mathrm{D}$ ions are taken into account. The flux limit factor for the parallel electron heat conductivity is taken to be 0.2 , consistent with the fluid-kinetic calculations in [13]. The ion heat flux is not fluxlimited, and the parallel viscous momentum flow is calculated from a 21 moment approximation [14] and limited to one half of the ion pressure. We did not vary the flux limit parameters, and discussion on the possible effect of such a variation is outside the scope of this paper. The cross-field transport coefficients are $\chi_{\perp \mathrm{i}}=\chi_{\perp \mathrm{e}}=1 \mathrm{~m}^{2} \mathrm{~s}^{-1}$ and $D_{\perp}=0.3 \mathrm{~m}^{2} \mathrm{~s}^{-1}$. These values result in a typical width of the power load profile of around $5 \mathrm{~mm}$ when mapped along the magnetic field onto the midplane, and in a radial pressure gradient in the midplane close to the experimental ballooning limit (see also [15]). There is no experimental evidence of steeper pressure gradients there, which can be considered an indirect justification of the selected values. The sensitivity of the solution to the $D_{\perp}$ and $\chi_{\perp}$ values has been addressed in [16], and it was found that reduction of these diffusivities by a factor 2 produced the same effect on the peak power loading as an increase of the input power by $25 \%$.

For this study, we have six series of runs: three values of $\xi_{\mathrm{ei}}=\frac{1}{3}, 1$, and 3 , and two fuelling scenarios-full core fuelling without gas puff $\left(\eta_{\mathrm{c}}=1\right)$ and low core fuelling $\left(\eta_{\mathrm{c}}=0.3-0.06\right)$. Figure 1 shows the variation of $q_{\mathrm{pk}}$ with the neutral pressure in the private flux region (PFR) $p_{\mathrm{DT}}=\Gamma_{\mathrm{DT}} / S_{\mathrm{DT}}$. All the data points lie on the same curve,

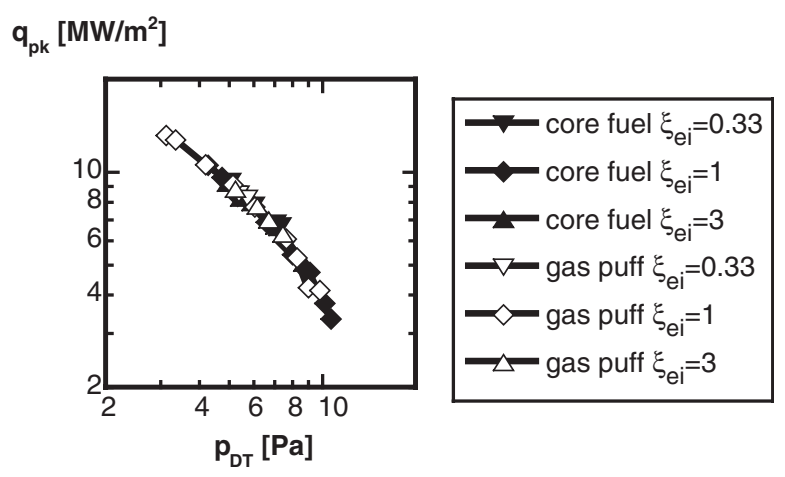

Figure 1. Peak power load on the target $q_{\mathrm{pk}}$ vs neutral pressure in the PFR $p_{\mathrm{DT}}=\Gamma_{\mathrm{DT}} / S_{\mathrm{DT}}$ for different fuelling scenarios and different values of the electron-to-ion power input ratio $\xi_{\mathrm{ei}}$.

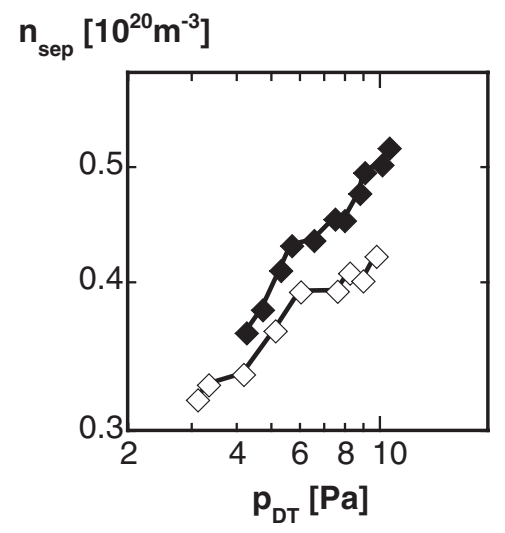

Figure 2. Separatrix-averaged density $n_{\text {sep }}$ vs $p_{\mathrm{DT}}$ for $\xi_{\mathrm{ei}}=1$ and different fuelling scenarios. Core fuelling is shown with solid symbols, and gas puffing with open symbols.

i.e. neither $\xi_{\mathrm{ei}}$ nor $\eta_{\mathrm{c}}$ affects $q_{\mathrm{pk}}$, and therefore the scaling for $q_{\mathrm{pk}}$ remains the same as in [4]. This is plausible, since energy equipartition in the divertor region is fast, and the particle flows are dominated by recycling fluxes which are much stronger than the throughput, so that the behaviour of the divertor plasma is insensitive to the details of energy and particle input to the edge.

However, the interface parameters, such as upstream densities and temperatures or neutral influxes, depend on the plasma parameters in the SOL, which depend on $\xi_{\mathrm{ei}}$ and $\eta_{\mathrm{c}}$. In figure 2 , the separatrix-averaged electron density $n_{\text {sep }}$ is plotted as a function of $p_{\mathrm{DT}}$ for different values of $\eta_{\mathrm{c}}$. No $n_{\text {sep }}$ saturation is seen for $\eta_{\mathrm{c}}=1$, although at the highest density, plasma in the inner divertor is already fully detached. There are two mechanisms that could explain the continued rise of $n_{\text {sep }}$ by the core fuelling. First, particles entering the SOL from the core must be transported along and across the magnetic field, and this requires a higher gradient, i.e. a higher $n_{\mathrm{s}}$, when the particle flux from the core increases (we distinguish here the separatrix density upstream $n_{\mathrm{s}}$ from the separatrix-averaged density $n_{\text {sep }}$; the ratio of $n_{\text {sep }} / n_{\mathrm{s}} \approx 1.1$ is approximately constant). For the gas-puffed case, the particles are mostly deposited in the outer part of the SOL and the density gradients in the separatrix region are smaller (figure 3(a)). Second, higher particle flux along the separatrix for the core-fuelled case enhances the convective transport of 
(a)

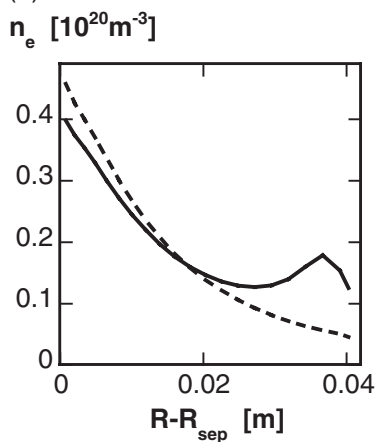

(b)

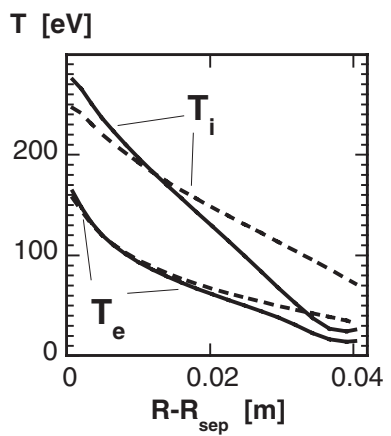

Figure 3. Radial profiles of (a) electron density $n_{\mathrm{e}}$ and $(b)$ electron and ion temperatures $T_{\mathrm{e}}$ and $T_{\mathrm{i}}$ for gas puffing $\left(\eta_{\mathrm{c}}=0.12,-\right)$ and core fuelling $\left(\eta_{\mathrm{c}}=1,---\right)$ cases. The data are averaged over magnetic surfaces, and the abscissa is the radial co-ordinate specified at the outer midplane.

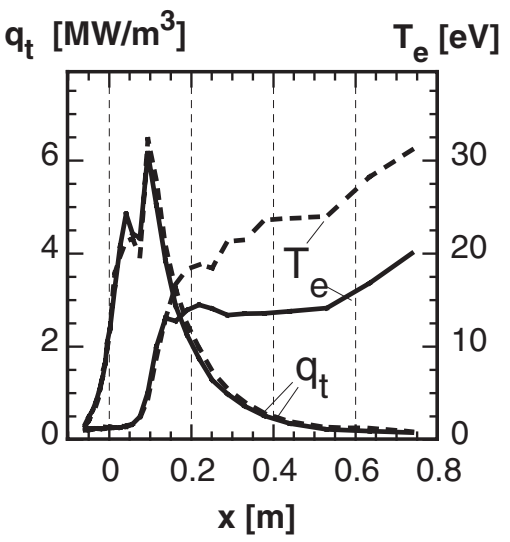

Figure 4. Profiles of power loading $q_{\mathrm{t}}$ and electron temperature $T_{\mathrm{e}}$ along the outer target for gas puffing $\left(\eta_{\mathrm{c}}=0.12, \longrightarrow\right)$ and core fuelling $\left(\eta_{\mathrm{c}}=1,-\right.$ - - $)$ cases. $x=0$ at the separatrix strike point, negative in the PFR.

energy, therefore reducing the conductive component. This results in a reduction of the upstream plasma temperature (figure $3(b)$ ) with corresponding increase-because of the pressure balance along the field-of $n_{\mathrm{s}}$. Moreover, the outer part of the SOL for the core-fuelled case becomes hotter and less dense, and this affects the profiles of plasma temperature along the target (figure 4).

A reduction of $n_{\text {sep }}$ when $\xi_{\text {ei }}$ decreases (figure 5), can be attributed to the different electron and ion heat conductivities. Indeed, an increase of the ion heat flux should cause an increase of the ion temperature upstream, which leads to a preferential increase of the conductive component of the flux due to the strong non-linear temperature dependence of the parallel heat conductivity, and the electron temperature changes in the opposite direction. The increase of the ion temperature is stronger than the reduction of the electron temperature because of the lower ion heat conductivity, so that lower $n_{\mathrm{s}}$ is required to satisfy the pressure balance. Since the convective component in the power flux is stronger when core fuelling is applied, the effect of the $\xi_{\text {ei variation is weaker }}$ there (figure $6(a)$ vs $(b)$ ).
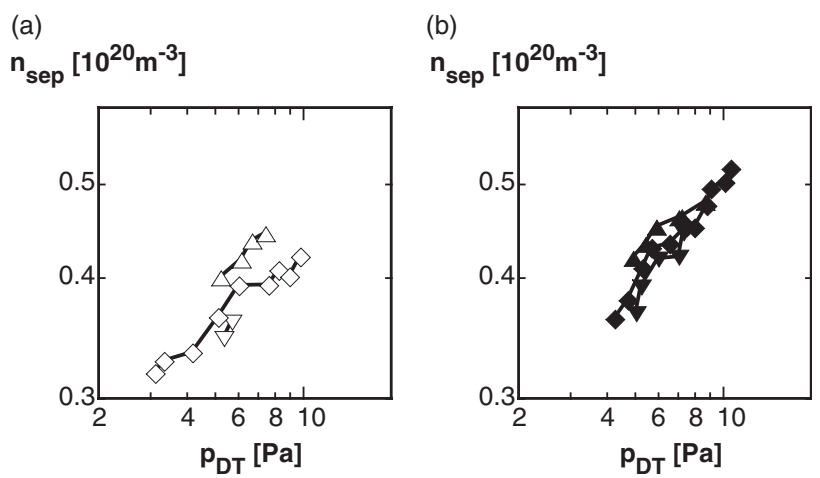

Figure 5. Separatrix-averaged density $n_{\text {sep }}$ vs $p_{\text {DT }}$ for different values of $\xi_{\mathrm{ei}}=P_{\mathrm{e}} / P_{\mathrm{i}}\left(\frac{1}{3}, 1\right.$, and 3$)$ for $(a)$ fuelling by gas puffing and (b) core fuelling. See legend of figure 1 . (a)

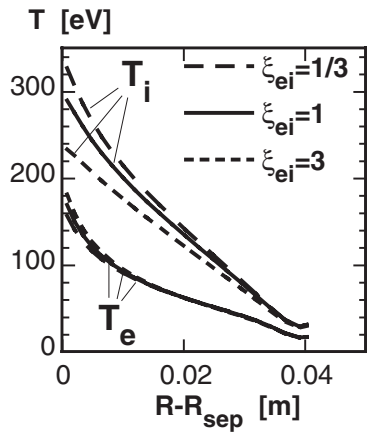

(b)

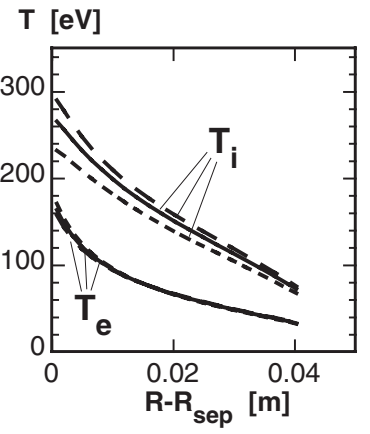

Figure 6. Radial profiles of electron and ion temperatures $T_{\mathrm{e}}$ and $T_{\mathrm{i}}$ for different values of $\xi_{\mathrm{ei}}=P_{\mathrm{e}} / P_{\mathrm{i}}$ for $(a)$ fuelling by gas puffing and (b) core fuelling. The data are averaged over magnetic surfaces, and the abscissa is the radial co-ordinate specified at the outer midplane.

\section{Scaling for the interface parameters}

A large number of modelling runs varying different parameters such as $\Gamma_{\mathrm{DT}}, P_{\mathrm{SOL}}, S_{\mathrm{DT}}, \xi_{\mathrm{ei}}$, and $\eta_{\mathrm{c}}$ have been carried out. To unify the results from these runs, we distinguish [4] two regimes of divertor operation, a non-saturated regime (regime a) at lower throughput and density and a saturated regime (regime b) at higher throughput and density. The variation of the transition point with the input parameters is determined and expressed in power law form. All quantities under consideration are normalized to their values at the transition point. It is then found that the multi-dimensional data fields can be reduced to single lines which can be fitted by a two-regime power law fit having different exponents below and above the transition (critical) value of density or throughput.

Following the approach of [4], we introduce the fuelling factor $f_{\mathrm{f}}=1+0.18 \eta_{\mathrm{c}}$, which expresses the fact that for ITER conditions the simulations show ion fuelling from the core to be $18 \%$ more effective in fuelling the SOL than neutral gas puffing from the edge. $P_{\mathrm{SOL}}$ is related to the fusion power via $Q$ and the core radiation fraction $f_{\text {rad }}$. The helium densities and fluxes quoted in the figures correspond to the core helium production rate for $Q=10$ and $f_{\text {rad }}=0.3$. Because helium-helium collisions are unimportant and the helium concentrations are low, helium fluxes and densities can be scaled to other values of $Q$ and $f_{\text {rad }}$ by applying the factor $f_{\mathrm{He}}=0.21(5 Q /(Q+5))\left(1-f_{\mathrm{rad}}\right)^{-1}$. 
It is found that these results can be unified in terms of the quantity $\mu=\left(\Gamma_{\#} / S_{\#}\right) P_{\#}^{-0.87} f_{\mathrm{f}}^{-2}$, i.e. the divertor neutral pressure (throughput over pumping speed) divided by an almost linear function of the SOL power and corrected by a function of the fuelling factor $f_{\mathrm{f}}$ which is close to 1 , with all quantities normalized to their value at the transition point (quantities normalized in this way are marked with a subscript '\#'). The maximum temperature at the inner divertor plate drops to a low value (figure 7) when this parameter exceeds 1.1 , i.e. the saturated regime $b$ is related to progressive plasma detachment at the inner divertor plate (see also [4]). Because experimental evidence shows a deterioration of the plasma confinement in the core when divertor detachment occurs [5], the higher density and throughput regime is less relevant and we therefore restrict our approximation in the following to the lower density regime for which $n_{\text {sep }}$ shows no sign of saturation (this applies also to the core-fuelled cases where saturation is not apparent). The maximum temperature along the inner divertor plate is twice as high for

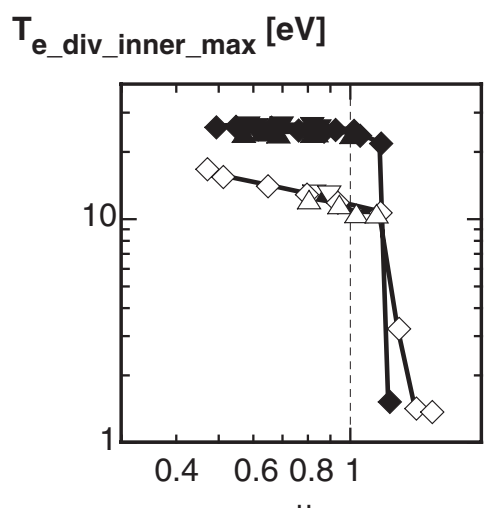

$\mu$

Figure 7. Variation of the maximum electron temperature along the inner target with the normalized neutral pressure $\mu=\left(\Gamma_{\#} / S_{\#}\right) P_{\#}^{-0.87} f_{\mathrm{f}}^{-2}$ in the PFR for core fuelling $(\boldsymbol{\diamond}, \boldsymbol{\Delta})$ and gas puffing $(\diamond, \triangle)$. See legend of figure 1 . the core-fuelled cases as for the cases with gas puffing. This can be attributed to the hotter periphery of the SOL in the case of core fuelling, as discussed above, and to the fact that the temperature maximum is located in this periphery (figure 4), as is typical for the vertical target configuration.

The exponents in the fitting expressions for the plasma parameters at the critical point where the two regimes meet are given in table 1, and for the plasma parameters at any working point in the non-saturated regime in table 2 . The normalization values ('scale') are given in both tables 1 and 2 . For example, at the critical point, $q_{\mathrm{pk} \#}=f_{\mathrm{f}}^{-1.7} P_{\#}^{1.26}$ where $q_{\mathrm{pk} \_}$is $q_{\mathrm{pk}}$ in units of $7.55 \mathrm{MW} \mathrm{m}^{-2}$ and $P_{\#}$ is $P_{\mathrm{SOL}}$ in units of $100 \mathrm{MW}$.

The quality of the fit is illustrated in figure 8 , where the output parameters are plotted according to the power law fit of table 2. The fit is seen to be quite good-the apparently poorer fit for the separatrix temperatures is due to the expanded ordinate (see the vertical scale in figures $8(e)$ and $(f)$ ). A fit for the carbon concentration at the separatrix will be produced once the conditions of carbon interaction with the original metal wall are modified in the two-dimensional modelling from the present assumption of the complete absorption to a more realistic model taking into account re-erosion of the deposited carbon.

The helium ion density at the separatrix and the helium neutral flux across the separatrix decrease strongly (table 2 and figure 8) as the $\mathrm{D} / \mathrm{T}$ throughput (and neutral pressure) increases. This effect is much stronger for the present (improved) helium modelling, which includes helium-D/T elastic collisions, than for previous simulations that did not include these, as further explained in [4]. As a result, the helium problem for ITER has been considerably alleviated and helium recycling no longer dominates the central helium concentration.

The peak power load at the divertor plate also decreases strongly (table 2 and figure 8 ) with neutral pressure in the divertor, and increases strongly with power. It should be noted that while the dependence on divertor pressure is plausible, it is non-trivial — the peak power load is made up of electron and

Table 1. Exponents in the parameter scaling at the critical point.

\begin{tabular}{|c|c|c|c|c|c|c|c|c|c|}
\hline & Scale & $q_{\mathrm{pk}}$ & $n_{\text {sep }}$ & $\Gamma_{\text {DT_n_sep }}$ & $n_{\mathrm{He} \_ \text {sep }}$ & $\Gamma_{\text {He_n_sep }}$ & $T_{\mathrm{e}_{-} \text {sep }}$ & $T_{\mathrm{i} \_ \text {sep }}$ & $\Gamma_{\mathrm{DT}}$ \\
\hline Scale & & $7.55 \mathrm{MW} \mathrm{m}^{-2}$ & $3.89 \times 10^{19} \mathrm{~m}^{-3}$ & 16.4 $\mathrm{Pa} \mathrm{m}^{3} \mathrm{~s}^{-1}$ & $3.06 \times 10^{17} \mathrm{~m}^{-3}$ & $0.512 \mathrm{~Pa} \mathrm{~m}^{3} \mathrm{~s}^{-1}$ & $162 \mathrm{eV}$ & $270 \mathrm{eV}$ & $124 \mathrm{~Pa} \mathrm{~m}^{3} \mathrm{~s}^{-1}$ \\
\hline$f_{\mathrm{He}}$ & 1 & - & 一 & - & +1 & +1 & - & - & - \\
\hline$f_{\mathrm{f}}$ & 1 & -1.7 & +1.25 & -2.5 & -5 & -5.42 & -0.4 & -0.9 & +2 \\
\hline$S_{\mathrm{DT}}$ & $20 \mathrm{~m}^{3} \mathrm{~s}^{-1}$ & - & - & +0.3 & -1 & -1 & -0.02 & -0.04 & +1 \\
\hline$P_{\mathrm{SOL}}$ & $100 \mathrm{MW}$ & +1.26 & +0.55 & - & +0.7 & 0.52 & +0.32 & +0.36 & +0.87 \\
\hline$\xi_{\mathrm{ei}}$ & 1 & - & +0.05 & - & -0.1 & - & +0.049 & -0.115 & - \\
\hline
\end{tabular}

Table 2. Exponents in the parameter scaling in terms of divertor pressure $p_{\mathrm{DT}}=\Gamma_{\mathrm{DT}} / S_{\mathrm{DT}}$ for the non-saturated regime (throughput below critical).

\begin{tabular}{|c|c|c|c|c|c|c|c|c|}
\hline & Scale & $q_{\mathrm{pk}}$ & $n_{\text {sep }}$ & $\Gamma_{\text {DT_n_sep }}$ & $n_{\text {He_sep }}$ & $\Gamma_{\text {He_n_sep }}$ & $T_{\text {e_sep }}$ & $T_{\mathrm{i}_{\text {_sep }}}$ \\
\hline Scale & & $7.55 \mathrm{MW} \mathrm{m}^{-2}$ & $3.89 \times 10^{19} \mathrm{~m}^{-3}$ & 16.4 $\mathrm{Pa} \mathrm{m}^{3} \mathrm{~s}^{-1}$ & $3.06 \times 10^{17} \mathrm{~m}^{-3}$ & $0.512 \mathrm{~Pa} \mathrm{~m}^{3} \mathrm{~s}^{-1}$ & $162 \mathrm{eV}$ & $270 \mathrm{eV}$ \\
\hline$f_{\mathrm{He}}$ & 1 & - & - & - & +1 & +1 & - & - \\
\hline$f_{\mathrm{f}}$ & 1 & - & +0.53 & -3 & -1 & -1 & -0.06 & -0.32 \\
\hline$S_{\text {DT }}$ & $20 \mathrm{~m}^{3} \mathrm{~s}^{-1}$ & - & - & +0.3 & -1 & -1 & -0.02 & -0.04 \\
\hline$P_{\mathrm{SOL}}$ & $100 \mathrm{MW}$ & +2 & +0.24 & -0.22 & +2.44 & +2.44 & +0.47 & +0.61 \\
\hline$\xi_{\mathrm{ei}}$ & 1 & - & +0.05 & - & -0.1 & - & +0.05 & -0.116 \\
\hline$p_{\mathrm{DT}}$ & 6.2 $\mathrm{Pa}$ & -0.85 & +0.36 & +0.25 & -2 & -2.21 & -0.17 & -0.29 \\
\hline
\end{tabular}




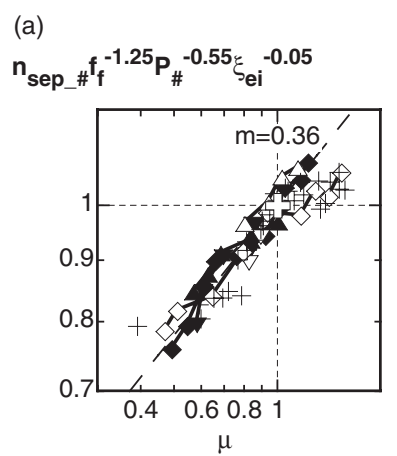

(c)

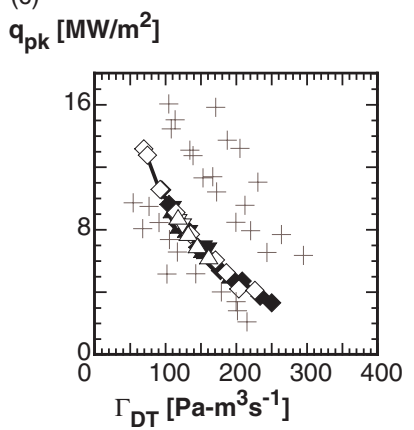

(b)

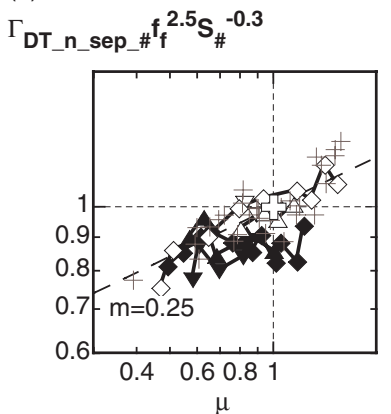

(d)

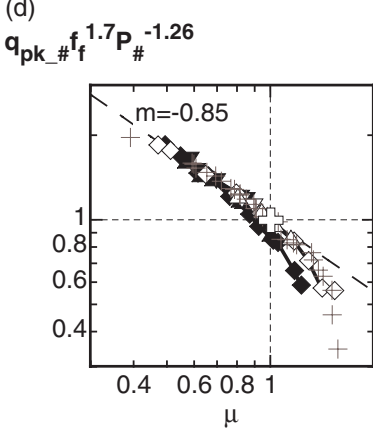

(e)

(f)

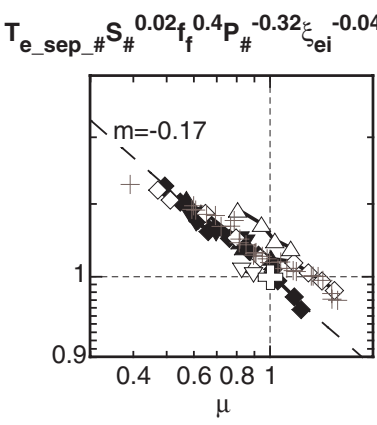

(g)

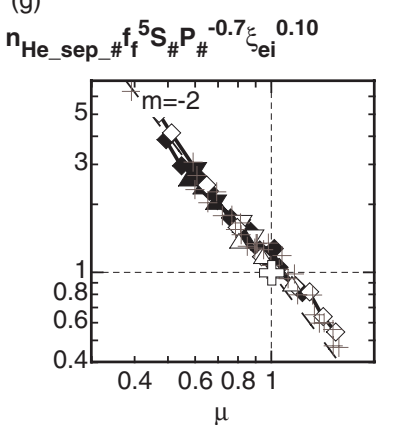

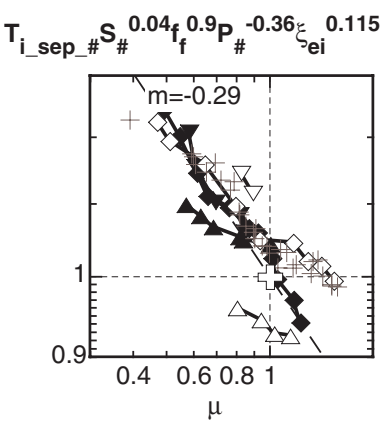

(h)

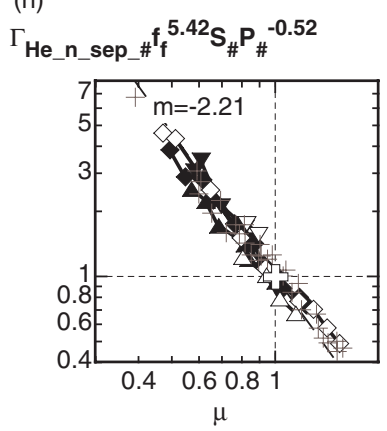

Figure 8. Fit to the separatrix-averaged values of ( $a$ ) density $n_{\text {sep }}$, (b) $\mathrm{D} / \mathrm{T}$ neutral influx to the core, $(d)$ peak power loading at the target, $(e)$ electron and $(f)$ ion temperatures, $(g)$ helium density $n_{\mathrm{He} \_ \text {sep }},(h)$ He neutral influx to the core. $(c)$ Gives an example of the raw, non-scaled data (peak power load). Helium quantities in $(g)$ and $(h)$ are for $Q=10$ and $f_{\text {rad }}=0.3$ (see text). The legend is the same as for figure 1 . The subscript '\#' indicates normalization by the constants of tables 1 and 2. The data from [4] for different values of the input power and pumping speed are shown with crosses. The hollow cross indicates the transition point between the two regimes. Scaling in the unsaturated regime is shown with a dashed line whose log-log slope, $m$, is indicated in the figures.

ion heat conduction, convection, recombination at the plate, and radiation, and all of these components are significant and have different profiles along the plate.

For ITER conditions, the D/T neutral flux across the separatrix (table 2 and figure 8 ) is small $\left(8-15 \mathrm{~Pa} \mathrm{~m}^{3} \mathrm{~s}^{-1}\right)$ and varies little, i.e. at higher throughput more $\mathrm{D} / \mathrm{T}$ neutrals are ionized in the SOL and the remaining neutral flux is similar. Because this neutral flux is so small and varies little with the plasma parameters, it is necessary to provide significant core fuelling, of the order of $80-100 \mathrm{~Pa} \mathrm{~m}^{3} \mathrm{~s}^{-1}$, to provide the density pedestal and the required core density [6-8].

Since charge exchange and elastic collisions drive the effective neutral temperature towards the ion temperature and the neutral mean free path is comparable to the width of the temperature profile, we recommend for the neutral temperatures at the separatrix that it be assumed that $E_{\mathrm{DT}_{-} \text {sep }}=T_{\mathrm{i}_{-} \text {sep }}$ and $E_{\text {He_sep }}=\min \left(T_{\mathrm{i}_{\text {_sep }}}, 60 \mathrm{eV}\right)$. The latter estimate follows from the peculiarity of the cross-section of the He elastic collisions with $\mathrm{D} / \mathrm{T}$ ions [17], which drops sharply at energies above $20 \mathrm{eV}$. The energy of $\mathrm{D} / \mathrm{T}$ atoms is not so important for the core model since they acquire the ion thermal energy after the first charge exchange in the 'pedestal' region where the density gradient is strong. A previous estimate, used in the ASTRA calculations of $[7,8]$, was the D/T neutral temperature $T_{\mathrm{n} \_ \text {sep }}=\frac{1}{2} E_{\mathrm{DT} \_ \text {sep }}=T_{\mathrm{i}_{\text {_sep }}} / 2$ and $E_{\mathrm{He} \_ \text {sep }}=30 \mathrm{eV}$.

\section{Effect of moving the strike-point position}

In order to check whether the limitation of the upstream density is determined by the $\mathrm{V}$-shaped target, we have performed several density scans using fuelling by gas puff, with upward displacements of the X-point from the standard position by $\Delta=6$ and $12 \mathrm{~cm}$ (see figure $9(a)$ ). A moderate upward shift of the X-point, $\Delta=6 \mathrm{~cm}$, results in an increase of the saturation density by $\sim 10 \%$. However, further increase of $\Delta$ to $12 \mathrm{~cm}$ leads to a bifurcation of the solution: at a certain neutral pressure in the PFR there is a discontinuous transition of all the output parameters towards another branch of the solution. In the course of the transition, a small decrease of particle throughput provokes a rapid (ms) redistribution of particles in the outer divertor, followed by a similar redistribution of particles in the inner divertor, all characterized by an increase of upstream density and a decrease of divertor pressure at constant SOL particle content, with corresponding increase of target loading by a factor 2 . The SOL particle content and divertor pressure then increase on a slow (tens of ms) timescale until the divertor pressure is again consistent with the particle throughput. The final state is on the low-pressure, high-density branch shown in figure 10. This bifurcation of the solution may be similar to the 'divertor MARFE' seen in the DIII-D experiment [18], but the theoretical model proposed there $[18,19]$ based on momentum transfer from the plasma flow in the divertor to the neutrals probably does not apply to the ITER case (different geometry, no volumetric recombination in that model), and another model based on the detail of the power transfer to the target [20] probably does not apply also for the same reasons. In any case, such a bifurcation is undesirable for divertor control, since small variations of the control parameter can lead to large excursions of power loading and core-edge interface parameters which are difficult to control. However, 


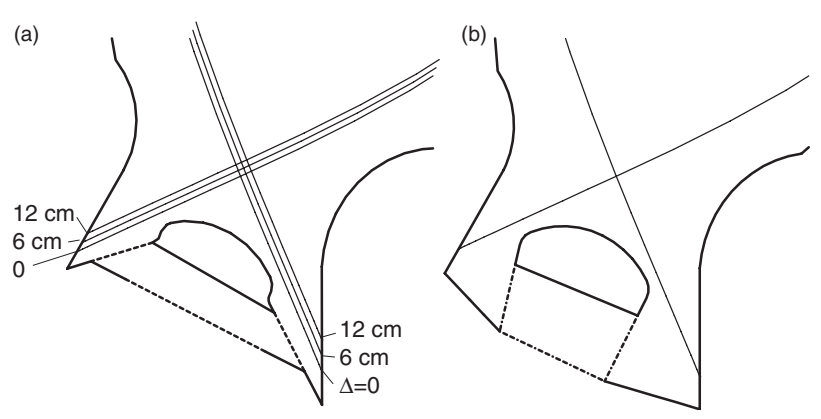

Figure 9. Variation of the divertor geometry used for the explorations: (a) the positions of the strike points marked with corresponding displacement of the X-point; $(b)$ the straight target geometry [1] (a)

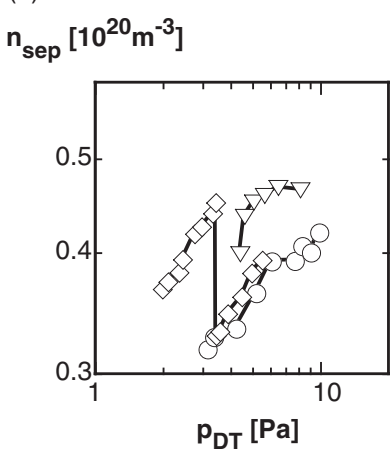

(c)

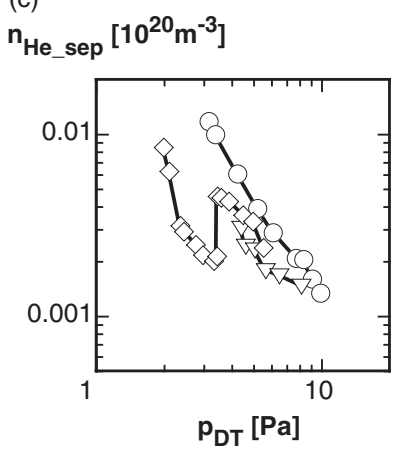

(a)

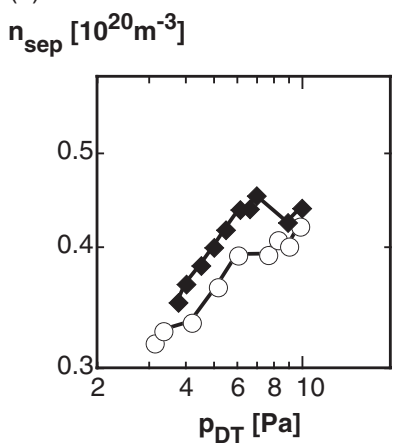

(b) $\mathrm{q}_{\mathrm{pk}}\left[\mathrm{MW} / \mathrm{m}^{2}\right]$

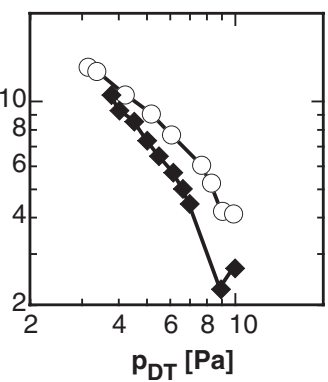

Figure 11. (a) Separatrix density and $(b)$ peak power vs divertor neutral pressure for the ITER target configuration ( $\mathrm{V}$-shape, $\mathrm{O}$ ) and the straight target option [1] ( $\mathbf{\square})$

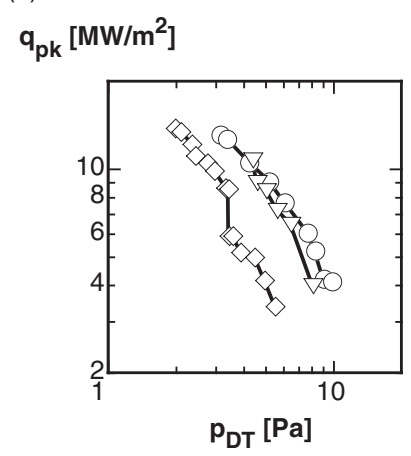

is shown in figure 11. Here, a density scan with gas puffing was performed for the straight target geometry of [1], modified to have the same finite gas conductance of the liners beneath the 'dome' as in the ITER design (figure $9(b)$ ). The input power is $100 \mathrm{MW}$ and the pumping speed $20 \mathrm{~m}^{3} \mathrm{~s}^{-1}$. The separatrix density for the straight target case reaches a value $\sim 15 \%$ higher than the density saturation level in the standard ITER geometry, and then a bifurcation appears which may be similar to that described earlier. At the highest density, plasma in the inner divertor detaches fully from the target. The peak power loading is lower than for the ITER geometry at the same throughput, but higher at the same separatrix density. Therefore, we believe that selection of the V-shaped target does not impose a fundamental limitation on the operational window of the ITER divertor.

\section{Toroidal effect in neutral transport}

A short exploration of the effect of including a more precise description of the toroidal geometry of ITER has been carried out to investigate refinements of the model. Up to now, all the calculations for ITER (e.g. [1-4]) have been done with a model that incorporates a description of transport in real toroidal geometry only for charged particles. The neutral transport was treated in the cylindrical approximation, since the neutral mean free path is much shorter than the major radius. However, some minor effects could be expected when the toroidal geometry is taken into account. First, the total recombination rate could be reduced by $\sim 10 \%$ since the major radius of the divertor in ITER is less than that of the plasma centre, and the latter was used for calculation of the cell volumes (and thus the total recombination source) in the cylindrical approximation. Similarly, the different major radii of inner and outer divertors can affect the relative parameters of the two divertors. Note that the recycling fluxes are calculated in the fluid part of the code, so that they are not affected by the approximation selected. The second possible effect is related to the presence of trans-sonic plasma flow downstream from the ionization front by partial detachment. In this case, there is a preferential direction in neutral scattering by charge exchange, causing a centrifugal effect on neutrals and affecting the particle exchange between the divertors.

In figure 12 , the results of a density scan (gas puffing, $P_{\mathrm{SOL}} \cong 100 \mathrm{MW}, S_{\mathrm{DT}}=20 \mathrm{~m}^{3} \mathrm{~s}^{-1}$ ) done in real toroidal 
(a)

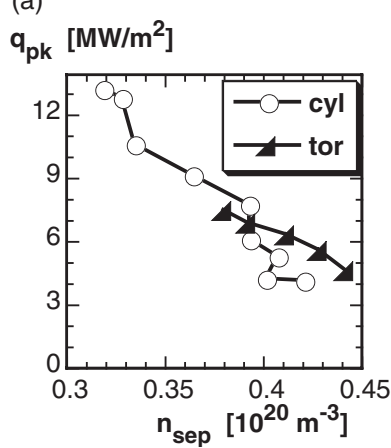

(b)

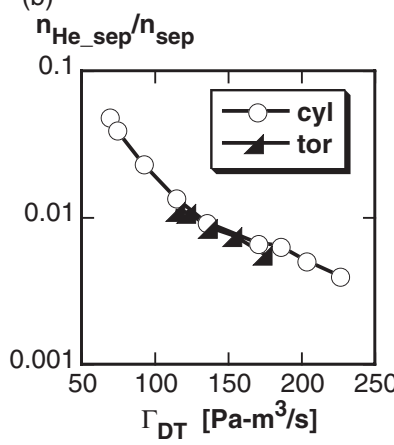

Figure 12. (a) Peak power vs separatrix density and (b) helium concentration vs D/T throughput, calculated using either the standard cylindrical approximation $(O)$ or real toroidal geometry for neutrals (solid symbols).

geometry are compared with those obtained with the standard cylindrical approximation for neutrals. It is difficult to judge the relative importance of the two kinds of toroidal effect discussed earlier, but the overall effect does not appear to be large. The peak power loading, the separatrix density and the helium exhaust efficiency are similar. Initial simulations (not yet fully converged) suggest that saturation and full detachment of the inner divertor may occur at higher throughputs for the toroidal calculation, i.e. the relative parameters of the two divertors are affected as noted above. This remains to be quantified.

\section{Conclusions}

The extensive modelling effort reported in this paper has led to an efficient power law parametrization of the plasma and neutral parameters at the interface between the edge and core plasma, making it finally possible to model the ITER core plasma performance in a way consistent with the divertor parameters [7, 8]. For a fixed geometry and transport properties, and without additional impurity seeding, five parameters largely determine the divertor performance. These are $P_{\mathrm{SOL}}$ - the power entering the SOL, $p_{\mathrm{DT}}$ - the neutral pressure in the divertor, $S_{\mathrm{DT}}$ - the pumping speed, $\eta_{\mathrm{c}}$ - the fraction of the core fuelling in the total $\mathrm{D} / \mathrm{T}$ particle throughput, and $\xi_{\mathrm{ei}}$ - the ratio of the electron to ion components of the $P_{\mathrm{SOL}}$. The interface plasma parameters are most sensitive to the $P_{\mathrm{SOL}}$ and $p_{\mathrm{DT}}$ values, whereas the $\xi_{\mathrm{ei}}$ value is relatively unimportant. The pumping speed, at constant divertor pressure, strongly affects the helium density upstream and helium atom influx to the core.

The core fuelling efficiency of gas puffing in ITER is expected to be low because of strong neutral screening by the hot, rather dense SOL, thus making core fuelling techniques such as pellet injection necessary for ITER.

The V-shaped target geometry employed in ITER does not impose additional constraints on the operational flexibility in terms of the achievable separatrix density.

A variation of the $\mathrm{X}$-point position offers some control over the achievable separatrix density. However, bifurcation of the divertor plasma parameters might limit the utility of this

control method. More work is needed to elucidate the exact nature of this bifurcation and its relevance to the performance of the ITER divertor.

Use of a cylindrical approximation instead of real toroidal geometry in the neutral modelling does not lead to large differences in the results so far. Specific aspects, such as the detachment of the inner divertor at high throughputs, may be different.

Further studies are under way to assess the effect of the divertor geometry, and to extend the scaling to cases having re-eroded carbon at the walls and/or additional impurity seeding.

\section{Acknowledgments}

This report was prepared as an account of work undertaken within the framework of ITER Coordinated Technical Activities (CTA). These are conducted by the Participants: Canada, the European Atomic Energy Community, Japan, and the Russian Federation, under the auspices of the International Atomic Energy Agency. The views and opinions expressed herein do not necessarily reflect those of the Participants to the CTA, the IAEA or any agency thereof. Dissemination of the information in this paper is governed by the applicable terms of the former ITER EDA Agreement, which continue to apply during the CTA.

\section{References}

[1] Kukushkin A.S. et al 2001 J. Nucl. Mater. 290-293 887

[2] Kukushkin A.S. et al 2002 Nucl. Fusion 42187

[3] Kukushkin A.S. and Pacher H.D. 2002 Plasma Phys. Control. Fusion 44931

[4] Pacher H.D., Kukushkin A.S., Pacher G.W. and Janeschitz G. 2003 J. Nucl. Mater. 313-316 657

[5] ITER Physics Basis 1999 Nucl. Fusion 39 2137-638

[6] Pacher G.W. et al 2001 Proc. 28th EPS Conf. on Control. Fusion and Plasma Phys. (Funchal, 18-22 June 2001) vol 25A (ECA) p 625

[7] Pacher G.W. et al 2002 Application of a 1-D predictive model for energy and particle transport to the determination of ITER plasma-SOL interface parameters Proc. 19th IAEA Fusion Energy Conf. (Lyon, France, October 2002) Paper FT/P2-11

[8] Pacher G.W. et al 2003 Nucl. Fusion 43188

[9] Reiter D., Wolf G.H. and Kever H. 1990 Nucl. Fusion 30 2141

[10] Reiter D. et al 1991 Plasma Phys. Control. Fusion 331579

[11] Schneider R. et al 1992 J. Nucl. Mater. 196-198 810

[12] Kukushkin A.S. et al 2003 Fusion Eng. Des. 65355

[13] Igitkhanov Yu.L., Kukushkin A.S. and Runov A.M. 1994 The impact of kinetic effects on the ITER divertor plasma Proc. 21st EPS Conf. on Control. Fusion and Plasma Phys. (Montpellier, 27 June-1 July 1994) vol 18B (ECA) p 766

[14] Bergmann A. et al 1996 Contrib. Plasma Phys. 36192

[15] Kukushkin A.S., Loarte A., Neuhauser J. and Sugihara M. 2000 Contrib. Plasma Phys. 40233

[16] Pacher H.D. et al 1999 J. Nucl. Mater. 266-269 1172

[17] Bachmann P. and Reiter D. 1995 Contrib. Plasma Phys. 35 2913

[18] Ghendrih Ph. et al 1995 J. Nucl. Mater. 220-222 305

[19] Ghendrih Ph. 1994 Phys. Plasmas 11929

[20] Krasheninnikov S.I. and Kukushkin A.S. 1988 Sov. Tech. Phys. Lett. 14228 